\title{
PCA based image denoising
}

\author{
${ }^{1}$ Y. Murali Mohan Babu, ${ }^{2}$ Dr. M.V. Subramanyam, ${ }^{3}$ Dr. M.N. Giri Prasad \\ ${ }^{1}$ Dept. of ECE, Sri Sai Institute of Technology and Science, Rayachoty, AP, India \\ ${ }^{2}$ Dept. of ECE, Santhiram Engineering College, Nandyal, AP, India \\ ${ }^{3}$ Dept. of ECE, JNTU College of Engineering, Anantapur, AP, India
}

\begin{abstract}
Principal component analysis (PCA) is an orthogonal transformation that seeks the directions of maximum variance in the data and is commonly used to reduce the dimensionality of the data. In image denoising, a compromise has to be found between noise reduction and preserving significant image details. PCA is a statistical technique for simplifying a dataset by reducing datasets to lower dimensions. It is a standard technique commonly used for data reduction in statistical pattern recognition and signal processing. This paper proposes a denoising technique by using a new statistical approach, principal component analysis with local pixel grouping (LPG). This procedure is iterated second time to further improve the denoising performance, and the noise level is adaptively adjusted in the second stage.
\end{abstract}

KEYWORDS: Principal component analysis, local pixel grouping, denoising, filter and discrete wavelet transform.

I. Introduction: PCA is a statistical procedure that uses an orthogonal property to transform to convert a set of observations of possibly correlated variables into a set of values of uncorrelated variables. The denoising phenomenon goal is to remove the noise while retaining the maximum possible the important signal or image features. At the time of acquisition and transmission the images are often corrupted by additive noise. The main aim of a denoising algorithm is to reduce the noise level, while preserving the image features. To achieve a good performance in this respect, a denoising algorithm has to adapt to image discontinuities. Generally the quality of image can be measured by the peak signal-to-noise ratio (PSNR). However, sometimes a denoised image with a high PSNR value does not have satisfactory visual quality [12].

PCA is a pre-processing transformation technique that creates new images from the uncorrelated values of different images [13]. This is accomplished by a linear transformation of variables that corresponds to a rotation and translation of the original coordinate system. PCA is used to findout principal components in accordance with maximum variance of a data matrix. Based on the principle components a new technique, based on maximization of SNR was also proposed in [3]. The grouping procedure guarantees that only the sample blocks with similar contents are used in the local statistics calculation for PCA transform estimation, so that the image local features can be well preserved after coefficient shrinkage in the PCA domain to remove the noise. Experimental results shows this method gives better performance, especially in image fine structure preservation, compared with general denoising algorithms [4-9]. 
Signal \& Image Processing : An International Journal (SIPIJ) Vol.3, No.2, April 2012

II. Median Filter: A median filter belongs to the class of nonlinear filters unlike the mean filter. The median filter follows the moving window principle like the mean filter. A $3 \times 3,5 \times 5$, or $7 \times 7$ kernel of pixels is scanned over pixel matrix of the entire image. The median of the surrounding pixel values in the window is calculated, and the center pixel of the resultant is computed and replaced with the computed median. Median filtering is done by, first sorting all the pixel values from the surrounding neighborhood into numerical order and then replacing the pixel being considered with the middle pixel value.

III. Lee Filter: This filter is an adaptive filter, which changes according to the local statistics of the current pixel. The Lee filter is based on the approach that if the variance over an area is low or constant, then the smoothing will be performed. Otherwise, if the variance is high, smoothing will not be performed.

IV. Gaussian filter: A gaussian filter is a filter whose impulse response is a gaussian function. Gaussian filters are designed to give no overshoot to a step function input while minimizing the rise and fall time. This behavior is closely connected to the fact that the Gaussian filter has the minimum possible group delay. Mathematically, a Gaussian filter alters the input signal by convolving with a Gaussian function.

V. Wiener filter: This approach often produces better results than linear filtering. This adaptive filter is more selective than a comparable linear filter, preserving edges and other highfrequency parts of an image. Wiener filters are a class of optimum linear filters which involve linear estimation of a desired signal sequence from another related sequence. The goal of the Wiener filter is to filter out noise that has corrupted a signal.

VI. Discrete Wavelet Transform: The Wavelet Transform provides a time-frequency representation of the signal. It was developed to overcome the previous method, Short Time Fourier Transform (STFT), which can also be used to analyze non-stationary signals. A timefrequency distribution of a signal provides information about how the spectral content of the signal evolves with time, thus providing an ideal tool to dissect, analyze and interpret nonstationary signals.

VII. Dual-tree complex wavelet transform: Dual tree complex wavelet transform (DT-CWT) has been developed to incorporate the good properties of the fourier transform in the wavelet transform. As the name implies 2 wavelet trees are used one generating the real part of the complex wavelet coefficients tree [10-11] and the other generating the imaginary part tree. The DTCWT comprises of 2 parallel wavelet filter banks trees that contain carefully designed filters of different delays that minimize the aliasing effects due to down sampling. The DTCWT of a signal is implemented using 2 critically sampled DWTs in parallel on the same data. Dualtree complex wavelet transform has many attractive properties, for example, approximate shift invariance, good directional selectivity and limited redundancy.

VIII. LPG-PCA denoising algorithm: Principal component analysis was developed by famous personalities the Pearson and the Hotelling, whilst the best modern reference is Jolliffe [2]. PCA is a method of identifying patterns in data, and expressing the data in such a way as to highlight their similarities and differences. Since patterns in data can be hard to find in data of 
Signal \& Image Processing : An International Journal (SIPIJ) Vol.3, No.2, April 2012

high dimension, where the luxury of graphical representation is not available, PCA is a powerful tool for analyzing data. The other main benefit of PCA is that once you have found these patterns in the data, and you compress the data, ie. by reducing the number of dimensions, without much loss of information.

Statistically, PCA is a de-correlation technique and it is mainly used in pattern recognition and dimensionality reduction and etc. By transforming the original dataset into PCA domain and preserving only the several most significant principal components, the noise and trivial information can be removed. A PCA-based scheme was proposed for image denoising by using a moving window to calculate the local statistics, from which the local PCA transformation matrix was estimated. PCA can be used to remove noise from images. PCA is a powerful statistical technique that is designed for determining lower dimensional representations of sampled data, which at first glance in its original representation may seem unstructured and random.

As shown in figure1, the proposed algorithm has two stages, in the The first stage it gives an initial estimation of the image by removing most of the noise and the second stage will further refine the output of the first stage [1]. The second stage has the same type of procedure except for the parameter of noise level. Since the noise in the first stage is significantly reduced, the LPG accuracy will be much improved in the second stage so that the final denoising result is visually much better.

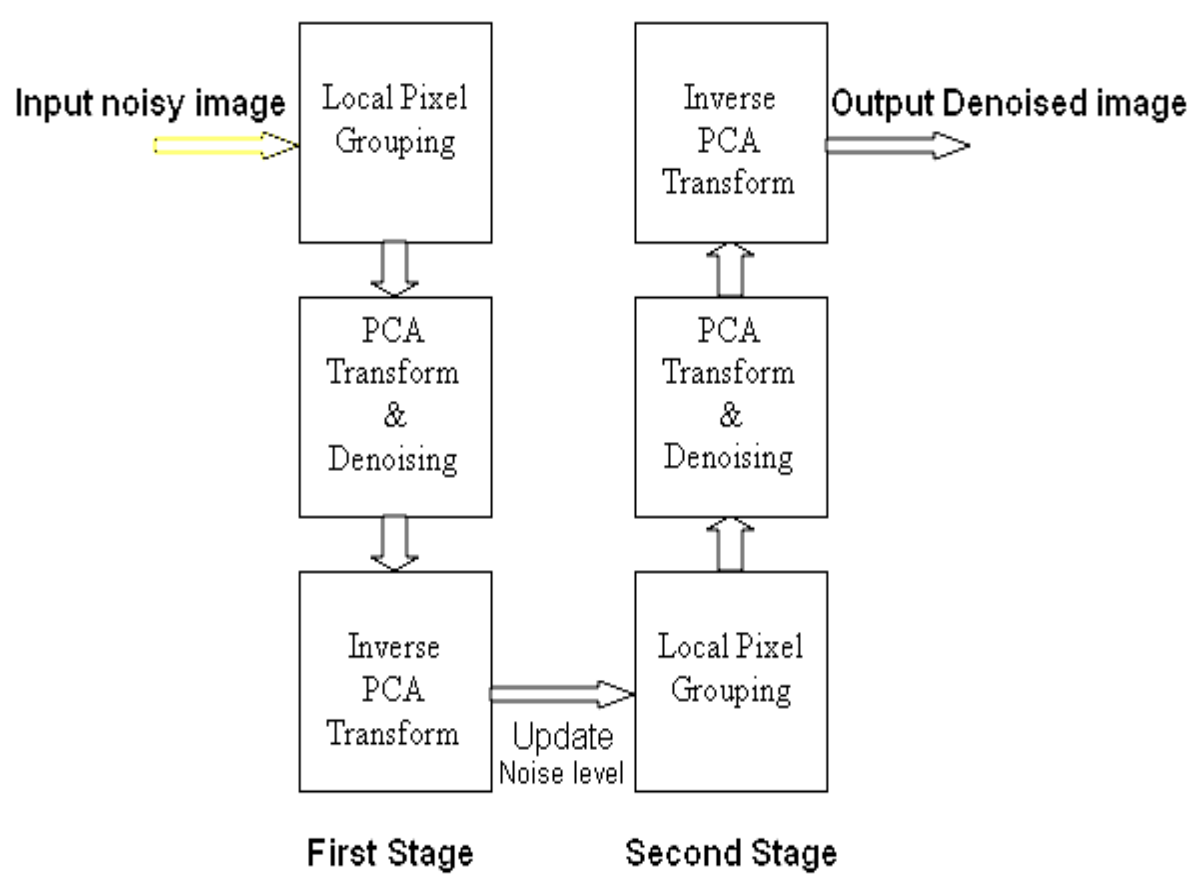

Figure 1:Two stage Principal component analysis

Thus we can implement the LPG-PCA denoising procedure for the second round to enhance the denoising results. 
Signal \& Image Processing : An International Journal (SIPIJ) Vol.3, No.2, April 2012

IX. Experimental results and discussions: Here we compared different denoising technologies like median filter, lee filter, weiner filter, gaussain filter, discrete wavelet transform, dual tree discrete wavelet transform and principle component analysis. Table1 shows the comparison of different denoising techniques for different test images. The two stage PSNR values have taken into consideration to compare.

It is clearly given that principle component analysis gives best PSNR value among all. The second stage got the better values compared to first stage. If the variance is high then second stage gives more signal-to-noise ratio values. For lower variance images first is sufficient to remove the noise.

Table.1: Comparison of PSNR values with different denoising techniques

\begin{tabular}{|c|c|c|c|c|c|c|c|}
\hline & City & Lenna & Monarch & Cameraman & Village & River & Average \\
\hline Median & 23.7841 & 22.9481 & 20.6154 & 20.8301 & 28.5012 & 22.5348 & 23.2022 \\
\hline Lee & 25.7401 & 25.7506 & 24.8241 & 25.6951 & 28.5655 & 24.6743 & 25.8749 \\
\hline Weiner & 25.6134 & 26.0118 & 24.6756 & 25.6588 & 29.6443 & 23.9915 & 25.9325 \\
\hline Gaussian & 26.7970 & 26.3957 & 25.2337 & 24.3735 & 29.3971 & 25.3792 & 26.2627 \\
\hline DWT & 28.1815 & 28.9758 & 28.0575 & 28.3796 & 31.2737 & 26.2009 & 28.5110 \\
\hline DT-DWT & 28.8201 & 30.1134 & 29.2085 & 29.0296 & 32.0129 & 26.5132 & 29.2829 \\
\hline PCA $-1^{\text {st }}$ Stage & 28.7660 & 30.2040 & 29.6746 & 29.5114 & 31.2017 & 26.8473 & 29.3675 \\
\hline PCA $-2^{\text {nd }}$ Stage & 28.7573 & 30.5415 & 30.0384 & 29.7184 & 31.5945 & 26.7205 & 29.5617 \\
\hline
\end{tabular}


Signal \& Image Processing : An International Journal (SIPIJ) Vol.3, No.2, April 2012

Table.2: Comparison of PSNR-1 \& PSNR-2 values for different images

\begin{tabular}{|c|c|c|c|c|c|c|c|c|}
\hline \multirow{2}{*}{} & \multicolumn{2}{|c|}{ City.tif } & \multicolumn{2}{c|}{ Village.png } & \multicolumn{2}{c|}{ Lenna.tif } & \multicolumn{2}{c|}{ River.png } \\
\cline { 2 - 9 } & PSNR-1 & PSNR-2 & PSNR-1 & PSNR-2 & PSNR-1 & PSNR-2 & PSNR-1 & PSNR-2 \\
\hline $\boldsymbol{\sigma}=\mathbf{5 0}$ & 24.1784 & 24.8051 & 26.2252 & 28.5672 & 24.8133 & 25.7227 & 23.2916 & 23.5307 \\
\hline $\boldsymbol{\sigma}=\mathbf{4 0}$ & 25.2845 & 25.6322 & 27.5460 & 29.2136 & 26.1654 & 26.8566 & 24.1530 & 24.1649 \\
\hline $\boldsymbol{\sigma}=\mathbf{3 5}$ & 25.9378 & 26.1675 & 28.2962 & 29.6198 & 26.9547 & 27.5468 & 24.6423 & 24.5817 \\
\hline $\boldsymbol{\sigma}=\mathbf{3 0}$ & 26.6939 & 26.8223 & 29.1270 & 30.1129 & 27.8594 & 28.3595 & 25.2034 & 25.1005 \\
\hline $\boldsymbol{\sigma}=\mathbf{2 5}$ & 27.6073 & 27.6555 & 30.0737 & 30.7416 & 28.9177 & 29.3343 & 25.8969 & 25.7758 \\
\hline $\boldsymbol{\sigma}=\mathbf{2 0}$ & 28.7660 & 28.7573 & 31.2017 & 31.5945 & 30.2040 & 30.5415 & 26.8473 & 26.7205 \\
\hline $\boldsymbol{\sigma}=\mathbf{1 5}$ & 30.3286 & 30.2897 & 32.6506 & 32.8287 & 31.8406 & 32.0964 & 28.3036 & 28.1741 \\
\hline $\boldsymbol{\sigma}=\mathbf{1 0}$ & 32.6523 & 32.6112 & 34.7685 & 34.7989 & 34.1299 & 34.2963 & 30.7099 & 30.6055 \\
\hline $\boldsymbol{\sigma}=\mathbf{5}$ & 36.9238 & 36.8965 & 38.6735 & 38.6163 & 38.0637 & 38.0966 & 35.4248 & 35.3854 \\
\hline $\boldsymbol{\sigma}=\mathbf{2}$ & 43.2961 & 43.2829 & 44.3663 & 44.2776 & 43.6649 & 43.6157 & 42.4872 & 42.4830 \\
\hline $\boldsymbol{\sigma}=\mathbf{1}$ & 48.6551 & 48.6536 & 49.2196 & 49.1426 & 48.6966 & 48.6810 & 48.2520 & 48.2520 \\
\hline
\end{tabular}

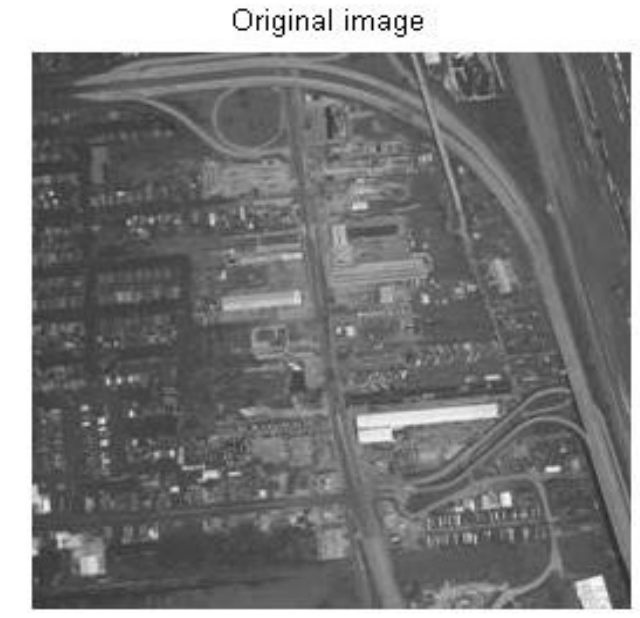

(I)

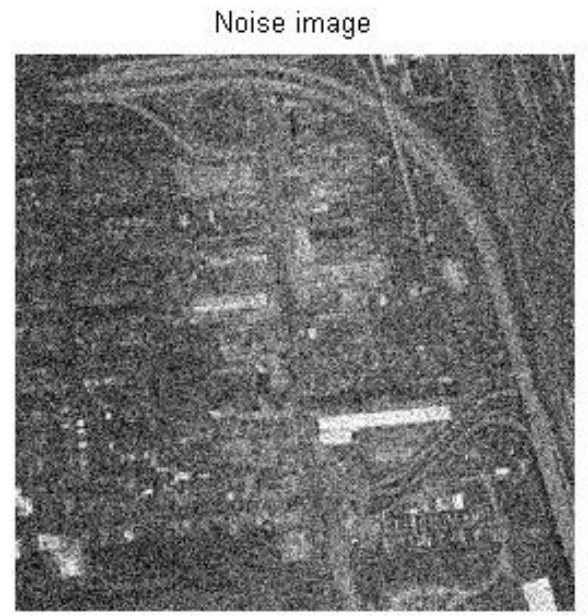

(II) 
Signal \& Image Processing : An International Journal (SIPIJ) Vol.3, No.2, April 2012

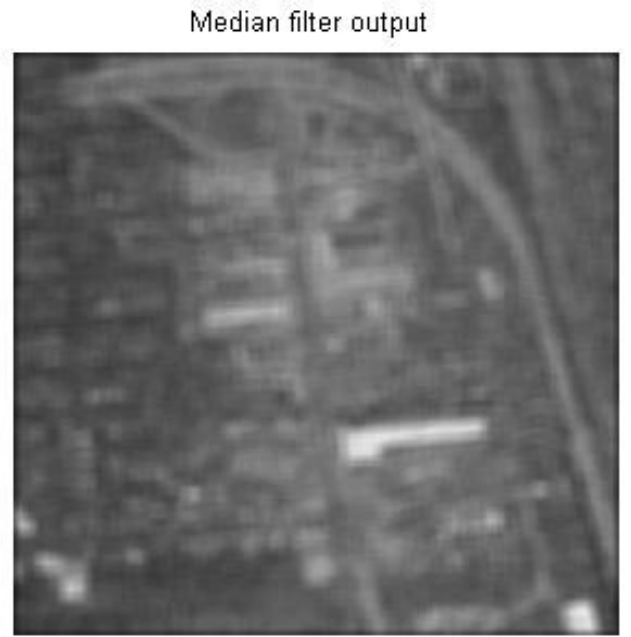

(III)

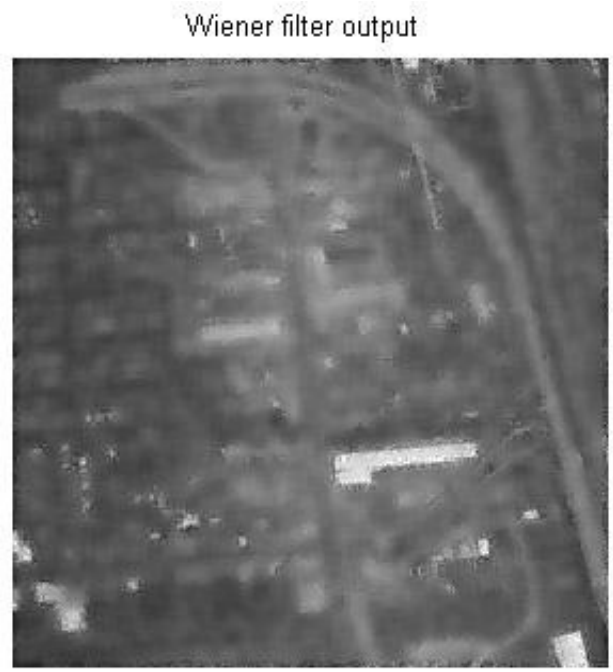

(V)

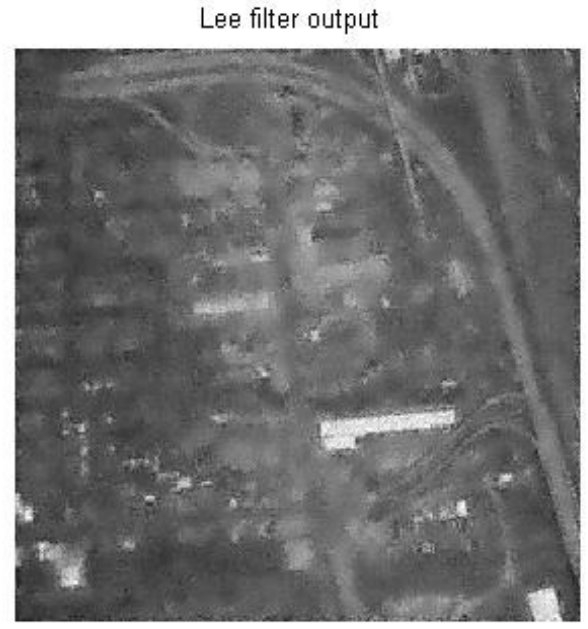

(IV)

Gaussian filter output

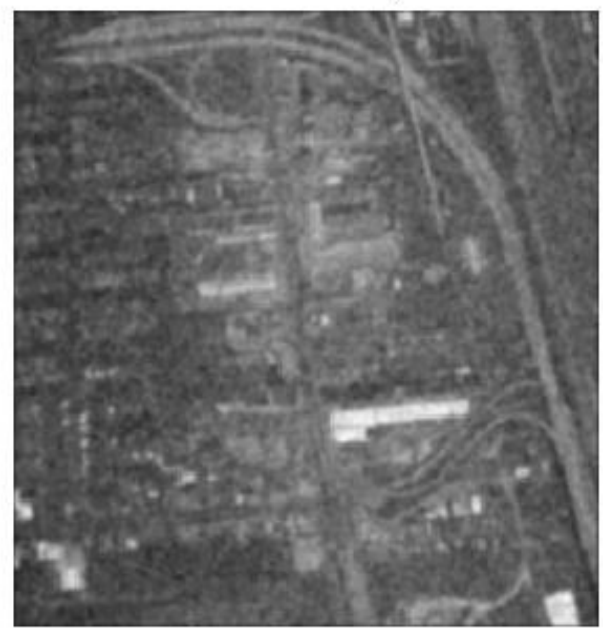

(VI) 
Signal \& Image Processing : An International Journal (SIPIJ) Vol.3, No.2, April 2012

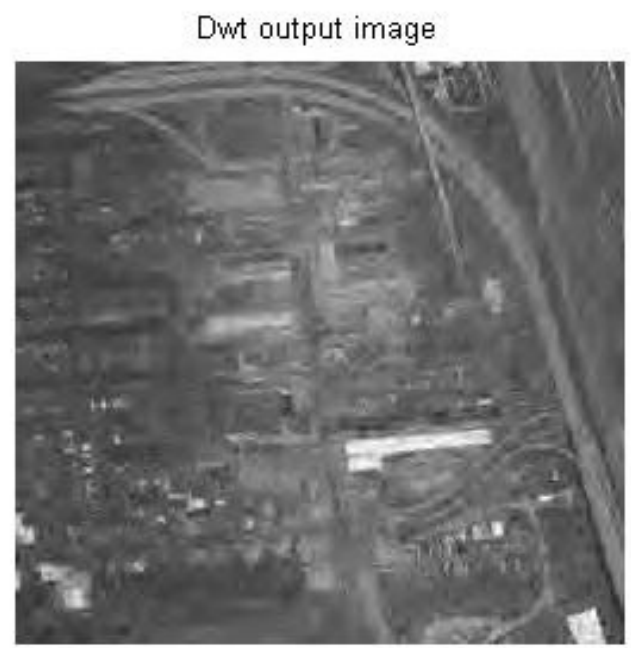

(VII)

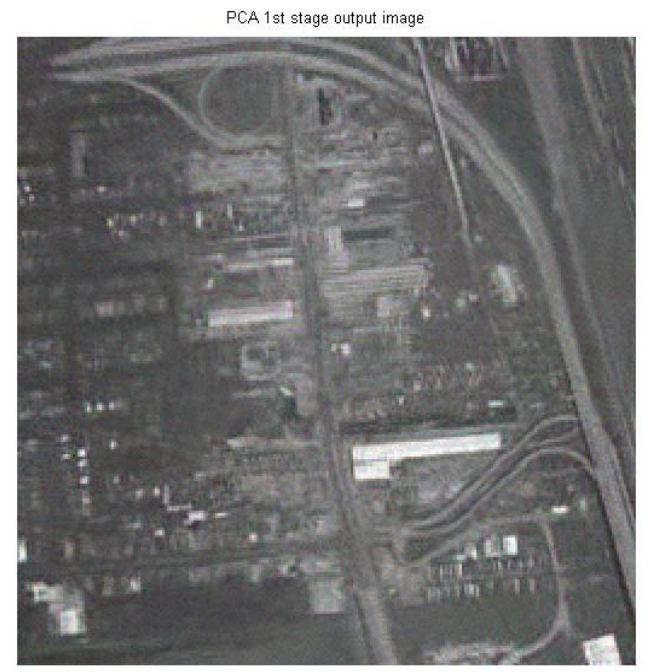

(IX)

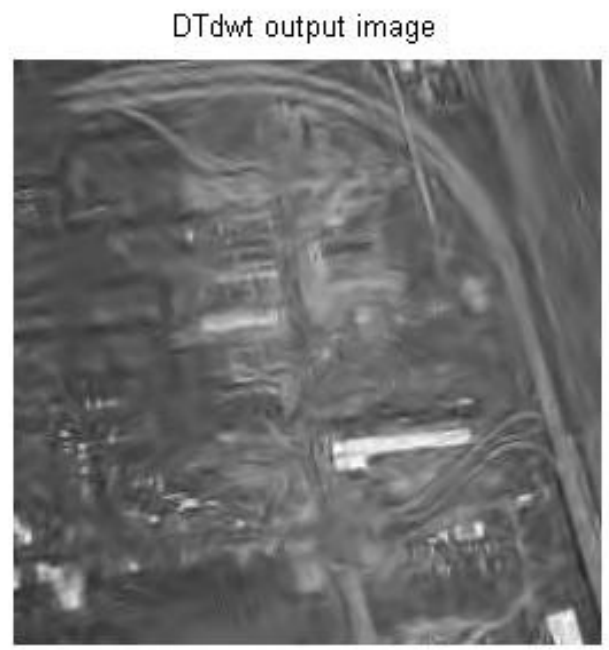

(VIII)

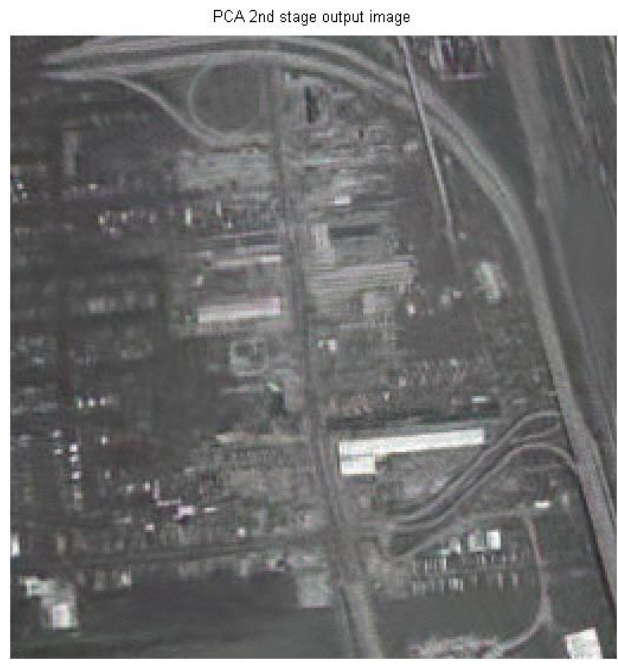

$(\mathrm{X})$

Figure2: (I)Original Image. (II)Noised Image. (III)Denoised image with median filter. (IV) Denoised image with lee filter. (V) Denoised image with weiner filter. (VI) Denoised image with gaussian filter. (VII) Denoised image with DWT. (VIII) Denoised image with DTDWT. (IX) Denoised image with $1^{\text {st }}$ stage of PCA. and (X) Denoised image with $2^{\text {nd }}$ stage of PCA. 
Signal \& Image Processing : An International Journal (SIPIJ) Vol.3, No.2, April 2012

\section{References:}

[1] Lei Zhang, Weisheng Dong, David Zhang, Guangming Shi, "Two-stage image denoising by principal component analysis with local pixel grouping" Elsevier-Pattern Recognition,vol-43, 2010, 1531-1549.

[2] Jolliffe, "Principal Component Analysis" Second ed. Springer Series in Statistics. New York: Springer-Verlag New York." 2002.

[3] Chein-I Chang, and Qian Du, "Interference and Noise-Adjusted Principal Components Analysis" IEEE transactions on geoscience and remote sensing, vol. 37, no. 5, sep. 1999,2387-2396.

[4] Y.MuraliMohanBabu, M.V.Subramanyam \& M.N.Giriprasad "Bayesian Denoising of SAR Image" International Journal of Computer Science \& Technology, Vol.2, Issue.1, PP-72-74, 2011,

[5] C. Boncelet, "Image noise models," in Handbook of Image and Video Processing, A. C. Bovik, Ed. New York: Academic, 2000.

[6] S. Mallat, "A Wavelet Tour of Signal Processing", 2nd ed. San Diego, CA: Academic, 1999.

[7] J. K. Romberg, H. Choi, R. G. Baraniuk, "Bayesian tree-structured image modeling using waveletdomain hidden Markov models," IEEETrans. Image Process., Vol. 10, No. 7, pp. 1056-1068, Jul. 2001.

[8] K. John Peter , Dr K.Senthamarai Kannan , Dr S. Arumugan , G.Nagarajan, "Two-stage image denoising by Principal Component Analysis with Self Similarity pixel Strategy”, International Journal of Computer Science and Network Security, VOL.11 No.5, May 2011, 296-301.

[9] Ayan Chakrabarti and Keigo Hirakawa, "Effective separation of sparse and non-sparse image features for denoising", ICASSP 2008, 857-860.

[10] Hadeel Nasrat Abdullah, Mohammed Fadhil Hasan and Qussy Salim Tawfeeq, "Speckle Noise Reduction in SAR images using double-density dual tree DWT" Medwell Journals, 2008,281-284.

[11] Hadeel Nasrat Abdullah, Mohammed Fadhil Hasan and Qussy Salim Tawfeeq, "SAR image denoising based on dual tree complex wavelet transform" Medwell Journals, 2008,587-590.

[12] Maryam Amirmazlaghani, Hamidreza Amindavar, "A Novelwavelet Domain Statistical Approach for Denoising SAR Images" ICIP, 2009.

[13] K. John Peter, Dr K. Senthamarai Kannan, Dr S. Arumugan , G.Nagarajan, "Two-stage image denoising by Principal Component Analysis with Self Similarity pixel Strategy", International Journal of Computer Science and Network Security, VOL.11 No.5, May 2011, 296-301. 
Signal \& Image Processing : An International Journal (SIPIJ) Vol.3, No.2, April 2012

\section{Authors}

Y. Murali Mohan Babu is working as Head and Associate Professor of the Electronics and Communication Engineering Department of Sri Sai Institute of Technology and Science, Rayachoty, Andhra Predesh, India. He has eleven years of teaching experience. He has done his B.Tech. Degree from JNTUniversity, Hyderabad, M.E. degree in Micro wave and Radar Engineering from Osmania University, Hyderabad. Currently he is pursuing his research work in Digital Image Processing in JNT University, Anantapur, AP, INDIA. His areas of interest are Communications, Signal Processing, Image Processing, Radar Systems and Microwave Engineering.

M.V.Subramanyam received his B.E degree in 1989, M. Tech degree in 1997 and $\mathrm{PhD}$ degree in 2007. Presently he is working as a 'Principal' and 'Professor' of 'Electronics and Communication Engineering' department of Santhi Ram Engineering College, Nandyal, Kurnool district, Andhra pradesh, India. He is having more than 21 years of teaching experience. His areas of interest are Adhoc Networks, Computer Networks, Advanced Communications, Signal Processing, Image processing, Embedded Systems and Microcontrollers. He has published more than 40 papers in national and international, conferences and international journals. He is a life member of ISTE, IEEE, IETE, IEI and KDTFM.

M. N. Giri Prasad received his B.Tech degree from JNTU College of Engineering, Anantapur, Andhra Pradesh, India in 1982, M. Tech degree from Sri Venkateswara University, Tirupati, Andhra Pradesh, India in 1994 and $\mathrm{PhD}$ degree from J.N.T University, Hyderabad, Andhra Pradesh, India in 2003. He is having more than 25 years of teaching experience. Presently he is working as Professor in the department of Electronics and Communication Engineering at JNTUA College of Engineering, Anantapur, Andhra Pradesh, India. His research areas are Wireless Communications, Biomedical Instrumentation, signal processing, image processing, embedded systems and microcontrollers. He has published more than 25 papers in national and international conferences. Around 50 papers published in national and international journals. He is a life member of ISTE, IEI and NAFEN. 